\title{
FORMACIÓN, ACTUALIZACIÓN Y PERFECCIONAMIENTO PROFESIONAL EN MATEMÁTICAS MODERNAS PARA DOCENTES DE LA ETAPA PRIMARIA DE EDUCACIÓN BÁSICA GENERAL
}

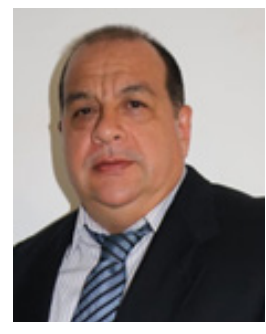

Martín Alexis Peralta Moreno ${ }^{1}$

Universidad Metropolitana de Educación, Ciencia y Tecnología, UMECIT,

Panamá

Universidad Tecnológica de Panamá, Departamento de Ciencias Exactas,

Panamá

mperaltam14@gmail.com

DOI: $10.37594 /$ oratores.n13.412

\section{RESUMEN}

La investigación se centró en describir las concepciones que tienen los maestros sobre las matemáticas modernas, su enseñanza y aprendizaje y, más concretamente, sobre su formación profesional en matemáticas, así como el conocimiento que poseen de las diferentes áreas que tienen que desarrollar según el programa del Ministerio de Educación. A partir de los datos suministrados por el diagnóstico y los análisis respectivos se diseñó una propuesta de formación, actualización y perfeccionamiento profesional en docentes de matemáticas modernas de la primera etapa de educación básica general, la cual se implementó y validó en escuelas de la comunidad de Betania, Distrito de Panamá. Se tomaron como informantes clave 8 docentes del área de matemática. El estudio se desarrolló bajo el enfoque cualitativo, a través del método hermenéutico interpretativo. La recolección de los datos se realizó a través de la técnica de la entrevista y la observación complementando con un análisis documental. Los resultados de este estudio revelaron que el conocimiento matemático de los maestros está circunscrito a las operaciones básicas con números naturales, influenciado por su concepción hacia la matemática clásica y la formación que recibió como estudiante de la licenciatura en educación. Se encontró que se utiliza un repertorio reducido de estrategias y técnicas para la enseñanza de las diferentes áreas de las matemáticas modernas. Esto es atribuible tanto a la formación profesional de cada docente como al currículo escolar cuyos nuevos contenidos, implantados por la llamada transformación curricular, los cuales no son de dominio explícito de los educadores que laboran en la educación primaria del nivel de premedia. A partir de este diagnóstico se diseñó, implementó y evaluó un programa de formación, actualización y perfeccionamiento para docentes de primaria con enfoques diferentes a los actuales, a la luz de las matemáticas modernas, centrados en cada una de las áreas establecidas en el programa curricular del Ministerio de Educación.

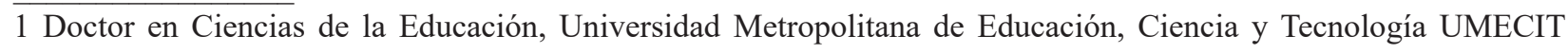
Panamá 
Palabras clave: Perfeccionamiento profesional, matemática moderna, educación primaria, programación analítica, categorización.

\section{TRAINING, UPDATING AND PROFESSIONAL ADVANCEMENT IN MODERN MATHEMATICS FOR TEACHERS OF THE PRIMARY STAGE OF GENERAL BASIC EDUCATION ABSTRACT}

This research focuses on describing teachers' conceptions of the teaching and learning of modern mathematics. More specifically, it looks at teachers' training in mathematics, as well as their knowledge of the different areas they are expected to teach according to the math program of the Ministry of Education. Based on analysis performed on diagnostic data, a proposal of professional development for math teachers working in grades first through sixth was presented. Implementation of this proposal was conducted in several schools in the Betania area in the district of Panama. Eight math teachers participated in a qualitative study that used the hermeneutic interpretation approach. Data was collected by means of interviews, observations and document analysis. The results of the study show that teachers' knowledge of mathematics is limited to basic natural number operations. This may be due to teachers' prevalent view of classical mathematics and their academic background in education school. Teachers used a limited repertoire of strategies and techniques to teach modern mathematics. This is partly due to teachers' own education as well as the content of the revised national curriculum of mathematics which is not fully mastered by teachers working in primary schools. As a result of this diagnostics, a professional development program which focuses on modern mathematics as it appears in the Ministry of Education's new curriculum aimed at elementary school math teachers was designed, implemented and evaluated.

Keywords: Primary education, modern mathematics, professional development, categorization, analytical programming

\section{INTRODUCCIÓN}

Los datos estadísticos proporcionados por el Ministerio de Educación sobre la Educación Panameña en el "Boletín No 1 Estadística Educativa: Final del año escolar 2012 MEDUCA 2013", tanto oficial como particular, no dejan la menor duda que nos encontramos inmersos en una crisis educativa en todos los niveles de nuestro sistema que nos conducen a la baja calidad de los aprendizajes, a elevados índices de fracasos, repitencia y deserción escolar.

El estudio Regional Comparativo y Explicativo (Serce 2006), evaluó en terceros y sextos 
grados de primaria las áreas de Matemática, Lectura y Escritura; Ciencias solamente se aplicó en sexto grado. El desarrollo de estas pruebas implicó la elaboración de un análisis curricular de los países de América Latina y el Caribe, a fin de establecer los dominios conceptuales y los procesos cognitivos comunes en dichos currículos.

Para Matemática, los saberes evaluados se referían al conocimiento y manejo de números y operaciones; del espacio y la forma; de las magnitudes y la medida; del tratamiento de la información y el estudio del cambio (secuencias, regularidades y patrones). Por su parte, los procesos cognitivos analizados comprendían el reconocimiento de objetos y elementos, y la solución de problemas simples y complejos.

El análisis posibilitó agrupar a los países en cinco categorías, de acuerdo con su diferencia respecto del promedio regional, y en donde los estudiantes panameños, tanto de tercer grado como de sexto grado, quedaron rezagados en la cuarta categoría con una puntuación media en Matemática inferior al promedio, solo por encima de Guatemala y República Dominicana.

El Ministerio de Educación de Panamá (MEDUCA) reveló los resultados del Tercer Estudio Regional Comparativo y Explicativo (TERCE). Esta investigación evaluó el aprendizaje de 15 países de América Latina y el Caribe en matemática, lectura y ciencias naturales, arrojando una conclusión clara para Panamá: el nivel de la educación es bajo y debe mejorarse. Por ejemplo, en matemática los estudiantes de tercer grado de primaria obtuvieron, en promedio, 664 puntos y los de sexto grado, 644 puntos. En esta asignatura el país mostró puntajes similares Nicaragua y Honduras, mientras que fue superado por Chile, Costa Rica, Uruguay, entre otros.

Moritz Bilagher, especialista de la Unesco en el tema, subrayó que el país debe prestar mucha atención a estas estadísticas. Precisó que se debe reforzar matemática en los alumnos de sexto grado y, lectura en los menores de tercer grado de enseñanza primaria.

Para Luis Montejano Peimbert (2013) presidente de la Sociedad Matemática Mexicana (SMM), "El sistema educativo está pervertido, pues aquellos maestros que odian las matemáticas le transmiten ese sentimiento a los niños y persiste la tradición de que esa materia es de lo peor, es lo más espantoso que uno pueda imaginar".

En el 2012, en la ciudad de Panamá, la Universidad Tecnológica de Panamá (UTP) realizó un sondeo en coincidencia con el Magisterio Panameño Unido a maestros de grados en ejercicios, encontrando entre muchos resultados que la enseñanza de las matemáticas en las escuelas primarias 
presenta deficiencias. El profesor Ricardo López, ex decano de la Facultad de Ciencias y Tecnologías de la UTP, que formó parte del estudio, indica que los maestros tienen fallas en geometría.

Según López (2013), esto se debe a que en el currículo académico de la Escuela Normal Juan Demóstenes Arosemena, en Santiago de Veraguas, no hay suficientes materias para que el maestro domine los conceptos a enseñar, pues demuestran bajo dominio en temas como perímetro, paralelogramos, tanto por ciento, operaciones con fracciones, entre otros.

El Doctor Eliécer Ching, en su momento decano de la Facultad de Ciencias y Tecnologías de la Universidad Tecnológica de Panamá (UTP), afirmó que las fallas en el aprendizaje de la matemática y el alto índice de fracasos en las escuelas básica general tienen efectos en las escuela media, y estos en la etapa universitaria. Puso de ejemplo que en los últimos años, en promedio de $54.6 \%$ de los aspirantes a ingresar a la UTP no alcanza el puntaje de aprobación en la prueba que se aplica como requisito de ingreso.

En el 2015 se realizó un sondeo a los profesores de matemáticas de los centros educativos Instituto Fermín Naudeau, Colegio Internacional de María Inmaculada, Instituto América y el Centro Educativo Dr. Octavio Méndez Pereira para conocer sus opiniones acerca del nivel académico en matemática con que llegan los alumnos a séptimo grado a estos colegios. Los resultados fueron casi unánimes al describir la incapacidad de los estudiantes en resolución de problemas, el desconocimiento de la temática de álgebra, el bajo conocimiento en geometría y el nulo conocimiento de probabilidades, lo que según ellos, los lleva a reprobar la asignatura desde inicio de año escolar pues sus bases académicas no son lo suficientemente sólidas para comprender la temática a desarrollar en séptimo.

Para el abordaje de la situación objeto de estudio fue necesaria una exploración previa de la problemática a través de la observación directa y entrevistas inestructuradas a un grupo de actores involucrados en la temática, que permitieron identificar síntomas concretos y evidencias de las debilidades y falencias existentes en los actuales currículos de formación de docentes en el área de matemáticas modernas. Esta situación no solamente tiene impacto en las instituciones de educación básica, sino que atiende también a una necesidad imperante de realizar revisiones y actualizaciones en los contextos de educación superior universitario frente a los planes de estudio y la necesaria correspondencia que debe existir con las exigencias del entorno, los emergentes cambios que son necesarios incorporar y de esa manera, aportar elementos que impacten en la acreditación de carreras a nivel superior y la pertinencia del perfil del egresado en el área de matemáticas modernas. 
La observación realizada permitió conocer de entrada que los docentes panameños especialistas en matemática y que atienden los grupos de séptimos grados de pre-media, afirman que la mayoría de los estudiantes llegan a estos grados con poco conocimiento de geometría y estadística, con una base muy débil en la aplicación de algoritmos aritméticos básicos, carentes de técnicas para la resolución de problemas matemáticos de la vida real y nulo conocimiento en principios de álgebra y probabilidades, necesarios para comprender y resolver adecuadamente los temas propuestos en la programación curricular del Ministerio de Educación, por lo que afirman, se dan muchos fracasos escolares en esta asignatura al ingresar a séptimo grado.

Esta situación motivó a realizar una investigación más precisa sobre el suceso que se ha estado presentando en los planteles educativos de primaria en la República de Panamá y poder de alguna forma conocer la raíz del problema, para presentar sugerencias de acciones y propuestas concretas con la finalidad de ayudar a encontrar la solución del mismo y hacer aportes que impacten en las políticas públicas en materia de educación, específicamente en la revisión y actualización de los currículos para la formación del docente en el área de matemáticas modernas.

Formar docentes con los requisitos actuales de una sociedad globalizada y competitiva, exige realizar cambios profundos en las instituciones formadoras de docentes y además, reafirmar la necesidad de evaluar integralmente la actual formación, de cara a implementar nuevos modelos de enseñanza y aprendizaje, que permita acceder a un nuevo modelo de educación.

Por todo lo expuesto se propuso como propósito analizar el impacto de un programa de formación, actualización y perfeccionamiento profesional en matemáticas modernas para docentes de la etapa primaria de la educación básica general.

\section{CONCEPTOS SENSIBILIZADORES}

\section{Formación de maestros}

La formación de maestros de grados es considerada muy deficiente por diversos colectivos dentro y fuera del sistema educativo. Entre las causas que hay que destacar las graves limitaciones del actual plan de estudios.

Rico (2000), denuncia el panorama desolador que se percibe en la formación matemática de los futuros maestros, "lo cual hace inteligible la preocupación social que se viene manifestando sobre la degradación de la enseñanza de las matemáticas en primaria, una de cuyas causas principales es la escasa y deficiente preparación de sus educadores" (p. 50). 
En este caso centramos la atención en la dimensión epistemológica (conocimientos matemáticos y didácticos), instruccional (tareas y patrones de interacción docente-discente) y competencial (desarrollo de competencias matemáticas). Otro rasgo característico de este proyecto es su naturaleza curricular y holística, en el sentido de que se propone desarrollar y experimentar documentos que abarquen la globalidad de contenidos matemáticos y didácticos que se consideran pertinentes para el ejercicio competente de la profesión de maestro en el área de matemáticas.

Cualquier cambio educativo está ligado a la formación del docente, o lo que es lo mismo, no hay transformación educativa sin transformación en la formación del maestro y profesor. Partiendo de esta idea, se hace evidente que si queremos cambiar la educación, si queremos adaptarla a la sociedad actual y a las necesidades de formación del alumnado, necesitamos cambiar la formación inicial del docente independientemente del nivel que ejerza.

\section{Supuestos epistemológicos, cognitivos e instruccionales de la propuesta curricular.}

Aquí se incluye una síntesis de los supuestos epistemológicos, cognitivos e instruccionales que orientan la propuesta curricular.

$>$ Las matemáticas como quehacer humano, lenguaje simbólico y sistema conceptual, donde se considerarán aspectos esenciales en la organización de su enseñanza:

- Las matemáticas establecen actividades de resolución de situaciones problemáticas de una cierta índole, socialmente compartida; estas situaciones problemáticas pueden referirse al mundo real y/o social o bien pueden ser internas a la propia matemática.

- Como respuesta o solución a esos problemas externos o internos aparecen y evolucionan progresivamente los objetos matemáticos (conceptos, procedimientos, teorías, ....).

- Las matemáticas son un lenguaje simbólico en el que se enuncian las situaciones problemas y las soluciones halladas; como todo lenguaje envuelve una serie de reglas las cuales hay que conocer y su aprendizaje ocasiona dificultades similares al aprendizaje de otro lenguaje no materno.

- Las matemáticas integran un sistema conceptual, lógicamente organizado y socialmente compartido; la organización lógica de los conceptos, teoremas y propiedades explican también gran número de las dificultades en el aprendizaje.

- La búsqueda de relaciones entre los diferentes objetos matemáticos coloca en juego los razonamientos inductivos y estimables, pero la estructuración de los resultados se realiza de acuerdo con la lógica deductiva. 
Conocer y aprender matemáticas, su relación con la resolución de problemas implica:

- Que la conceptualización del conocimiento matemático, "conocer" o "saber" matemáticas, por una persona, no puede limitarse a identificar las definiciones y propiedades de los objetos matemáticos. Debe ser capaz de usar el lenguaje y el sistema conceptual matemático en la resolución de problemas y emplear constructivamente el razonamiento matemático.

- La actividad realizada con el fin de resolver problemas es uno de los pilares del aprendizaje significativo de las matemáticas, además de una fuente de motivación intrínseca hacia la misma, ya que permite contextualizar y personalizar los conocimientos.

- Que los estudiantes deben tener oportunidad de investigar sobre problemas a su alcance, formular conjeturas, probar, construir modelos, lenguajes, conceptos, teorías, intercambiar sus ideas con otros, reconocer las que son conformes con la cultura matemática, adoptar las ideas que le sean útiles.

El trabajo del educador es producir una contextualización y personalización de los conocimientos, ya que debe buscar las mejores situaciones que den sentido a dichos conocimientos y ayudar al estudiante en la búsqueda de las soluciones, las que se convertirán en sus propios conocimientos.

\section{Criterios para la formación matemática y didáctica de maestros.}

La formación de los futuros maestros de primaria en elárea de las matemáticas debe concentrarse en los conocimientos profesionales sobre la enseñanza y el aprendizaje de las matemáticas del nivel educativo correspondiente. No obstante, el estudio de los problemas didácticos no es posible sin un conocimiento suficiente del contenido disciplinar al que se refieren dichos conocimientos didácticos, en nuestro caso los contenidos matemáticos presentes en los programas curriculares de primaria propuestos por el Ministerio de Educación de Panamá.

El núcleo básico de la didáctica de las matemáticas sobre contenidos impartidos en los primeros niveles educativos es la construcción del sentido del lenguaje, los conceptos y métodos matemáticos por parte de los niños, mediante su referencia a las situaciones y problemas matemáticos presentes en la vida cotidiana. Esta atribución de significado a las tareas matemáticas escolares requiere conocimientos y destrezas matemáticas por parte del educador que con frecuencia no están disponibles para los futuros maestros, y por tanto se deben contemplar en el período de su formación inicial. 
Estas estimaciones conllevan a considerar como materia troncal en los planes de formación de maestros especialistas en matemática una asignatura curricular que recibe el nombre de "Matemáticas y su didáctica".

El análisis didáctico-matemático de los problemas, situaciones y tareas matemáticas para la educación primaria, deben ser el eje central de la formación del maestro desde el área de la Didáctica de la Matemática, partiendo de la selección y estudio de problemas y dificultades que den sentido a los conceptos y métodos matemáticos propuestos en el currículo. Esto permitirá contextualizar las nociones teóricas de didáctica que se consideren pertinentes como herramientas de análisis de los procesos de enseñanza y aprendizaje.

En los capítulos siguientes presentamos, en líneas generales, los criterios seguidos en el proyecto para la formación y actualización de maestros en especialidades matemáticas y un manual de matemáticas y su didáctica. Esta propuesta, como se explicará más adelante, está abierta a la reflexión, experimentación, y progresivo enriquecimiento entre el colectivo de formadores de maestros.

\section{Capacitación y perfeccionamiento (formación en servicio)}

Para el ejercicio de la profesión docente se requiere capacitación en servicio y desarrollo profesional. La primera está relacionada con la mejora en el ejercicio docente, con la práctica diaria en el salón de clases y la segunda con las oportunidades para que los educadores puedan seguir progresando en otras áreas de su desarrollo profesional. Los sistemas de alto desempeño capacitan a los maestros en las escuelas, envían profesores experimentados para que den entrenamiento específico, particularmente a los maestros de bajo desempeño, lo que requiere un proceso de evaluación para conocer las debilidades y reforzarlas.

La capacitación docente o formación docente se refiere a las políticas y procedimientos planeados para preparar a potenciales profesores dentro de los ámbitos del conocimiento, actitudes, comportamientos y habilidades, cada uno necesario para cumplir sus labores eficazmente en la sala de clases y la comunidad escolar.

Para el Ministerio de Educación de Panamá, el propósito integral de las acciones de capacitación y perfeccionamiento es colaborar con el mejoramiento de la calidad de la educación, a través de la formación y la actualización profesional del personal en servicio. En los albores de la modernización educativa, se exige pensar en una formación docente que permita desarrollar y aumentar las fortalezas y capacidades diferenciales a favor de la formación integral general y 
especial, la actualización y la profesionalización de la docencia. Se pretende que los educadores sean ante todo diseñadores de métodos y de ambientes de aprendizaje que compartan en equipo con los estudiantes.

\section{RECORRIDO METODOLÓGICO}

El presente estudio se desarrolló bajo un enfoque cualitativo, ya que se centró en conocer y comprender un problema educativo desde la perspectiva de los actores involucrados, a partir de la interrelación del investigador con las posturas, criterios, opiniones y experiencias de los docentes de primaria en ejercicio de la profesión, con respecto a su formación, comprensión y aplicación de las matemáticas modernas en el aula de clases. Se analizaron además los planes de estudios de la Licenciatura en Educación Primaria, comparando, porcentualmente, el número de cursos y de créditos de matemática con respecto al total de la oferta académica. También se hizo un estudio minucioso del contenido de las diferentes áreas que se contemplan en la asignatura de matemática, planteadas en los programas curriculares de quinto y sexto grado, propuestos por el Ministerio de Educación para las escuelas primarias.

Sentados estos argumentos, nos apoyamos en el método hermenéutico-interpretativo para guiar la investigación, el cual ayudó a concentrar el interés del estudio en la interpretación y comprensión profunda de la realidad plasmada en los cuestionarios, tomando en cuenta el contexto y como consecuencia propiciar una construcción subjetiva del conocimiento que respalde y dé sentido a la realidad investigada.

Con la metodología detallada se consiguió interpretar el fenómeno de estudio, estimando y valorando aquellas observaciones, percepciones, pensamientos y reflexiones expresadas por las personas como derivaciones de las experiencias personales y profesionales, y tomando distancia de toda idea preconcebida. En el desarrollo de esta investigación se analizaron varios casos únicos a la vez, para estudiar la realidad que se deseaba explorar, describir, explicar, evaluar y modificar; el abordaje se realizó en dimensiones equivalentes para todas las instituciones y la comparación constante pretendió producir teoría partiendo del cruce de contextos. Esto concuerda con la orientación epistemológica del carácter hermenéutico-interpretativo adoptado.

Para lograr la participación de los informantes y obtener la descripción más exacta posible se implementando en ellos la entrevista semiestructurada, el cuestionario abierto, la observación directa y la indagación documental, como complemento que permite el análisis de la situación objeto de estudio desde una lógica mucho más integral. 
La triangulación de métodos, que sugiere contrastar las informaciones recogidas con diferentes técnicas, fue la estrategia que permitió clasificar, comparar y relacionar las opiniones de los docentes informantes plasmadas en la entrevista semiestructurada, con los resultados del cuestionario abierto, con las observaciones realizadas por el investigador y con la revisión documental de los programas académicos para la formación de docentes de primaria, con la finalidad de obtener información correspondiente con el tema de investigación.

Se realizó un análisis sobre la estructura programática de los cursos que conforman el plan de estudio del Programa de la Licenciatura en Educación de la Universidad de Panamá, del Instituto Pedagógico Superior Juan Demóstenes Arosemena y los del ISAE universidad para identificar las características y representatividad de la asignatura matemática en la organización curricular de dichos programas. Estos Programas de las carreras de Licenciatura en Educación Primaria fueron proporcionados por las propias instituciones formadoras de docentes.

\section{RESULTADOS}

A partir de los antecedentes recopilados en el Marco Teórico y de los datos obtenidos en la investigación, se establecieron las categorías y subcategorías con el fin de poder realizar comparaciones y posibles contrastes, de manera que se pueda organizar conceptualmente los datos y presentar la información siguiendo algún tipo de patrón, haciendo con ellos la siguiente lista:

\section{Tabla $\mathrm{N}^{\circ} 1$}

Categorías y subcategorías para el registro de la información, según criterio del investigador, año 2015

\begin{tabular}{|c|c|}
\hline Categorías & Subcategorías \\
\hline $\begin{array}{l}\text { Características de la formación } \\
\text { matemática del docente de primaria. }\end{array}$ & $\begin{array}{l}\text { - } \text { Teórica } \\
\text { - } \text { Práctica } \\
\text { - } \text { Enfóco-Práctico } \\
\text { aplicados tradicionalmente enseñados y } \\
\text { - Competencias, habilidades y destrezas que se } \\
\text { desarrollan. }\end{array}$ \\
\hline $\begin{array}{l}\text { - Debilidades encontradas en } \\
\text { los contenidos de los cursos de } \\
\text { matemáticas para formar docentes de } \\
\text { primaria. }\end{array}$ & 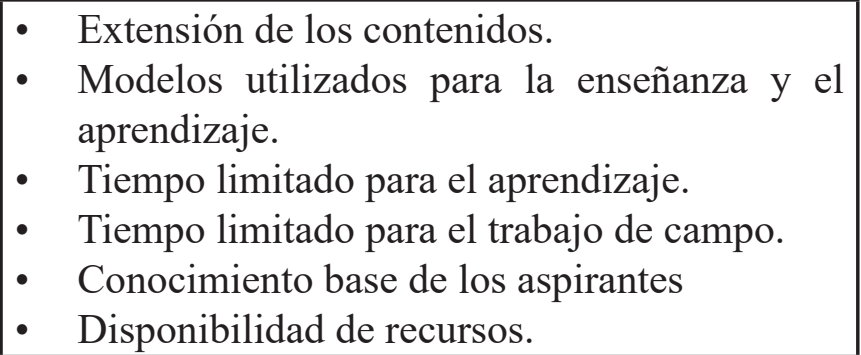 \\
\hline
\end{tabular}




\begin{tabular}{|l|ll|}
\hline - $\begin{array}{l}\text { Orientación de los cursos de } \\
\text { matemáticas en el aula de clases. }\end{array}$ & $\bullet \begin{array}{l}\text { Curricular } \\
\text { Estrategias metodológicas y técnicas }\end{array}$ \\
\hline $\begin{array}{l}\text { Percepción de los educadores sobre } \\
\text { su formación y capacitación para } \\
\text { desarrollar clases de matemáticas } \\
\text { modernas en el ejercicio profesional } \\
\text { como docentes. }\end{array}$ & - $\begin{array}{l}\text { Pertinencia } \\
\text { Aplicabilidad } \\
\text { Consistencia teórica-práctica }\end{array}$ \\
$\begin{array}{l}\text { Necesidaddeintroduciractualizaciones } \\
\text { y capacitaciones de matemáticas } \\
\text { modernas en sus diferentes áreas. }\end{array}$ & $\begin{array}{l}\text { Comprensión de las nuevas áreas de las } \\
\text { matemáticas modernas contempladas en los } \\
\text { actuales programas educativos. } \\
\text { Desarrollo de la capacidad para producir y } \\
\text { hacer producir conocimientos matemáticos. } \\
\text { Valoración del entorno como medio y objeto } \\
\text { de estudio matemático. }\end{array}$ \\
\hline
\end{tabular}

Fuente: El autor del estudio

Las categorías y subcategorías fueron construidas antes y durante el proceso recopilatorio de la información, que surgieron desde el levantamiento de referenciales significativos a partir de la propia indagación.

"Programa de formación, actualización y perfeccionamiento profesional en matemáticas modernas para docentes de la etapa Primaria perteneciente al nivel de Educación Básica General”, como complemento correspondiente de los cursos que se dictan en las instituciones educativas que ofrecen la Licenciatura en Educación Primaria, bajo convenios suscritos entre el Ministerio de Educación y la Universidad de Panamá.

Basados en la revisión meticulosa de las fuentes teóricas referidas al tema de la formación profesional de docentes para educación primaria, en la opinión expresada por los informantes del estudio y en las interpretaciones, reflexiones y aportes del autor, detalladas en las conclusiones y recomendaciones, se plantea la siguiente propuesta con la finalidad de actualizar y fortalecer la formación en matemáticas modernas de los docentes que laboran y laborarán en la etapa primaria de la Educación Básica General en la República de Panamá.

\section{Aspectos generales de la propuesta}

Esta propuesta está compuesta por dos acciones de mejoras, una consiste en la reestructuración del Diseño Curricular de los cursos Matemática I y II, que constituyen las asignaturas del plan de estudio de la Licenciatura en Educación Primaria que se imparte en las instituciones formadoras 
de docentes, y la otra es la Programación Analítica de los cursos necesarios para la actualización y perfeccionamiento profesional de los docentes en servicio sobre las áreas sensitivas de las matemáticas modernas complementarias a las de su formación académica.

\section{CONCEPTUALIZACIÓN Y PERTINENCIA DE LA PROPUESTA}

La UNESCO (2013) a través de la Oficina Regional de Educación para América Latina y el Caribe (OREALC), publicó el informe "Antecedentes y Criterios para la Elaboración de Políticas Docentes en $A L C$ " en donde manifiesta la débil calidad de los programas y de los procesos de formación inicial del docente, y lo sustenta así: "existe una duda generalizada respecto a la calidad de la oportunidad para aprender a enseñar que ofrecen las instituciones formadoras ... La duda por la calidad se sustenta, sobre todo, en los resultados de las evaluaciones estandarizadas de aprendizaje de los alumnos y alumnas a lo largo del sistema escolar, y también, en los resultados de la participación en evaluaciones internacionales"

En pleno siglo XXI el educador debe ser consciente de las necesidades de nuevas habilidades que implica su rol docente, y que su tarea cambió de transmitir conocimientos a ayudar a aprender, y que debe perseguir su continuo crecimiento profesional para tener un apreciado desempeño en su quehacer educativo. En esta dirección el maestro debe conocer y utilizar el contenido matemático a enseñar de manera amplia, de modo que le permita realizar su función educativa con seguridad y adaptarse a los nuevos cambios curriculares si es necesario.

Esta propuesta se estructura a partir de las siguientes consideraciones:

$>$ La transformación curricular implementada en el sistema educativo panameño trajo consigo la inclusión de nuevas áreas en la asignatura de matemática en la etapa primaria del nivel de la Educación Básica General, lo que ha reformado las labores y necesidades de los maestros de estos grados provocando un bache entre lo aprendido en su formación académica superior y lo requerido en esta innovación curricular.

$>$ La sociedad actual demanda cada vez más que los centros escolares guíen y formen niños y jóvenes capaces de interpretar, razonar y aprender a lo largo de su vida, esto se logra desarrollando pensamientos lógicos matemáticos, por lo que los docentes requieren evidenciar el dominio de estos conocimientos y competencias, de habilidades fundamentales y procesos de razonamientos superiores.

$>$ La variabilidad de contextos sociales y educativos reclaman el uso de otras metodologías para abordar los nuevos temas de matemáticas insertados en los programas de la etapa primaria para interpretarlos, comprenderlos y explicarlos lo cual sugiere la necesidad de ensayar nuevas alternativas para alcanzar la comprensión de los actores educativos. 
Los cursos de matemática, en los programas de estudio superiores de la Licenciatura en Educación Primaria, continúan con los mismos contenidos que antes de la transformación curricular implementada por el MEDUCA, lo cual indica que la formación en matemática de los nuevos docentes permanecerá igual, distante de las necesidades y exigencias actuales en nuestras aulas escolares.

\section{SISTEMATIZACIÓN DE LA PROPUESTA}

A raíz del problema estudiado en este trabajo de investigación se advirtió la necesidad que tienen los docentes de educación primaria de una preparación más razonable e íntegra con respecto a las áreas de las matemáticas modernas, ya que los actuales son insuficientes con respecto a los desafíos que tienen que enfrentar hoy día en las aulas escolares.

De allí que la propuesta de intervención a implementar para dar solución al problema investigado está compuesta de dos posibles actuaciones, y estas a su vez, de dos acciones necesarias concretas, a mencionar:

A. La primera actuación tuvo como objetivo encaminar, en las áreas de matemáticas modernas, a los docentes de primaria que están ejerciendo funciones académicas. En esta se planteó la Programación Analítica de dos Seminarios-Taller "Teórico-Procedimental" en donde se desarrollan los nuevos contenidos, incluidos por la transformación curricular, con estrategias metodológicas y técnicas específicas para comprenderlos y aprender a enseñar estos temas. Estas acciones fueron:

$\checkmark$ Seminario-Taller: Estrategias Metodológicas para la Enseñanza de los Números Enteros y del Álgebra a Nivel Primario.

$\checkmark$ Seminario-Taller: Estrategias Metodológicas para la Enseñanza de la Geometría, la Estadística y las Probabilidades a Nivel Primario.

B. La segunda actuación se centró en complementar los cursos universitarios actuales referentes a la asignatura de matemática. En esta se planteó la inclusión de las áreas, contenidos y temas necesarios que deben dominar los estudiantes de Licenciatura en Educación Primaria de las diferentes universidades. Para ello se tomaron como referentes los nuevos temas incluidos en la transformación curricular y así poder distribuirlos adecuadamente en la Programación Analítica de Asignatura en su respectivo curso universitario. Estas acciones fueron:

$\checkmark$ Programación Analítica de: Matemática Básica, Mat 203

$\checkmark$ Programación Analítica de: Geometría, Mat 214 
Cabe resaltar que el Seminario-Taller: Estrategias Metodológicas para la Enseñanza de los Números Enteros y del Álgebra a Nivel Primario, fue desarrollado en la Escuela Octavio Méndez Pereira, con el permiso otorgado por la Directora Regional de Educación de Panamá Centro y con el respaldo de 14 de los 23 docentes de la sección primaria, con una duración de una semana, otorgándoles el certificado de 40 horas.

El desarrollo del seminario, así como su asistencia fue supervisada por personal de la Dirección de Perfeccionamiento Profesional del Ministerio de Educación, ya que la propuesta se le hizo directamente a ellos con la finalidad de promover este seminario a nivel nacional para el próximo periodo vacacional o para el momento que ellos decidan sea conveniente.

Los documentos oficiales utilizados en el desarrollo de este seminario, como el pre test, post test, lista de asistencia y otros, se encuentran en los anexos de este trabajo de investigación.

La evaluación por parte de los docentes fue absolutamente satisfactoria, ya que solicitaban al MEDUCA que se programaran más seminarios como estos, en donde se desarrollan temas que no dominan y a los cuales deben enfrentarse en las aulas escolares. Además, solicitaban seminarios de las otras áreas de las matemáticas, pues su dominio no alcanza la totalidad de los nuevos contenidos programáticos incluidos por la transformación curricular.

La Dirección Nacional de Perfeccionamiento Profesional del Docente nos comunicó en carta membretada la satisfacción de la implementación de este seminario en la Escuela Octavio Méndez Pereira, por lo que será tomado en cuenta para las próximas planificaciones generales a nivel nacional.

\section{IMPLEMENTACIÓN DE LA PROPUESTA}

Se logró la implementación del programa Seminario-taller: "Estrategias Metodológicas para la Enseñanza de los Números Enteros y del Álgebra a Nivel Primario” en la Escuela Octavio Méndez Pereira, contando con el permiso oficial de la Dirección Regional de Educación de Panamá Centro y del revisado por la Dirección Nacional de Formación y Perfeccionamiento Profesional. Este seminario fue desarrollado la primera semana de vacaciones estudiantiles comprendida del 6 al 10 de junio de 2016. Fue supervisado directamente por el director encargado de la escuela el magíster Gustavo Macías. De los 23 maestros, asistieron 14 la jornada y semana completa.

El primer día se implementó un pre-test elaborado con contenidos conceptuales y procedimentales sobre los temas de números enteros y de álgebra, que deberían saber ellos para 
poder desarrollar sus clases de matemáticas. Al recoger el pre-test se dio la oportunidad de expresar los sentimientos, las percepciones y su disposición a la necesidad de empoderarse de los nuevos y exigentes aprendizajes sobre los contenidos de las matemáticas modernas.

Posteriormente, en cada jornada se realizó un trabajo arduo en la comprensión de conceptos matemáticos esenciales, de las reglas o leyes de signos y de las operaciones fundamentales con números enteros y con expresiones algebraicas. Se trabajó en talleres individuales y en equipos para conocer e implementar métodos, técnicas y actividades que favorecieran la comprensión de los estudiantes. Realizaron presentaciones magistrales y sustentaciones explicativas procedimentales utilizando los conocimientos adquiridos o recordados.

Antes de finalizar la última jornada, se aplicó la misma prueba del pre-test con la finalidad de poder evaluar el alcance e impacto que había tenido el desarrollo del seminario en los educadores participantes.

El asombro fue mayor para ellos al darse cuenta que en esta segunda oportunidad podían identificar, resolver y comprender en su totalidad las interrogantes que tenía dicha prueba.

Al dar paso a los comentarios, la mayoría de los participantes manifestaron su satisfacción por el nivel de comprensión adquirido en la resolución de operaciones y problemas de aplicación con números enteros y con expresiones algebraicas. Todo esto fue reflejado en el documento de evaluación del seminario aplicado por las autoridades del Ministerio de Educación.

\section{DE LOS DATOS A LA TEORÍA}

La teoría social trata de un conjunto de teorías de menor generalidad que describen aspectos parciales dentro del amplio espectro que abarcan las ciencias sociales. El concepto de teoría que aquí nos interesa, concibe a la teoría como una construcción intelectual racional que ordena, describe o explica en forma resumida un conjunto de hechos o fenómnos aislados (Ziman, 1984, Campbell, 1992).

El objetivo fundamental de la ciencia es la construcción de teorías, las cuales pueden ser producto de la intuición, la experiencia o de la investigación metódica. Kuhn (1962), afirma que las teorías, más que la simple investigación, han sido y son fuente de las mayores revoluciones científicas.

Lo importante es tener presente que la teoría ideal y necesaria es aquella que es novedosa, 
explicativa, amplia, pertinente, coherente, sencilla, comprobable y estimulante de nuevos descubrimientos.

En lo concerniente a este trabajo de investigación, el objeto y problema de estudio fue, si la formación académica universitaria de los docentes de primaria, específicamente con respecto a las matematicas modernas, es suficiente para desarrollar los nuevos programas propuestos por la transformación curricular realizada por el Ministerio de Educación de Panamá.

A través de las diferentes técnicas implementadas en cada fases de la investigación se pudo comprobar que:

$>$ Es bajo el nivel de comprensión que los maestros tienen con respecto a las matemáticas modernas (evidenciado en la entrevista).

$>$ Las metodologías y las técnicas son siempre las mismas, hay poca innovación en el desarrollo de las clases a la hora de enseñar matemáticas (observación directa)

$>$ Disconformidad por la formación matemática recibida en la universidad, ya que sienten que sus docentes no les enseñaron a enseñar matemáticas (entrevista)

> Actitud no muy satisfactoria a la hora de enseñar matemáticas modernas, pues su poco dominio le impide desplegar con motivación lo que desea enseñar (entrevista)

$>$ Los cursos actuales de matemáticas para formar docentes de educación primaria son pocos y sus contenidos se quedaron desfasados en comparación con las necesidades que trajo la transformación curricular.

Por otro lado el Ministerio de Educación no ofrece, a los educadores de la sección primaria, las acciones necesarias para nivelar ese vacío que existe entre lo que el docente sabe y lo nuevo que necesita saber, según la transformación curricular.

Todo esto tiene a los docentes de educación primaria, especialmente a los que laboran con los grupos de quinto y sexto grado, en una incertidumbre e inseguridad al momento de desarrollar sus clases, pues sienten que no lo están haciendo bien, o no lo están haciendo como debería ser.

Ahora, al implementar el Seminario-Taller: "Estrategias metodológicas para la enseñanza de los números enteros y del álgebra a nivel primario" a los docentes de la escuela El Japón, nos percatamos de la gran cantidad de interrogantes, así como de confusiones que ellos tenía, inclusive con las operaciones elementales con matemáticas básicas. Al terminar dicho seminario se pudo obtener opiniones de satisfacción y de gratitud por parte de los participantes, donde comunicaban que "no se puede enseñar bien algo que no se sabe ni se domina bien". 
Formación, actualización y perfeccionamiento profesional en matemáticas modernas para docentes de la etapa primaria de educación básica general Martín Alexis Peralta Moreno (27:50)

Uniendo todas estas partes del proceso de investigación, se sustenta la tesis la cual afirma que:

"Los resultados en matemática de los estudiantes de educación primaria de quinto y sexto grado no mejorarán significativamente si no dotamos a sus docentes del conocimiento conceptual y procedimental de las matemáticas modernas contempladas en los programas de la transformación curricular”, Martín Peralta (2017).

El siguiente mapa conceptual representa las asignaturas que contempla el programa actual de sexto grado para la escuela primaria en la República de Panamá. En la asignatura de Matemática se indican las diferentes áreas que se deben desarrollar, así como el contenido en cada una de ellas. La Programación Analítica de las dos propuestas identificadas como seminario-taller que se proponen hará impacto directamente en el conocimiento de los contenidos conceptuales y procedimentales que se resaltan en rojo, ya que estos no están contemplados en las asignaturas de matemática que reciben en la Licenciatura en Educación Primaria o fueron las que más sobresalen con mayor dificultad de comprensión por parte de los informantes.

Cabe resaltar que esta propuesta contempla la aplicación de diferentes métodos, técnicas y actividades para el desarrollo en cada una de las jornadas, orientando a los docentes en la comprensión y apropiación de los conocimientos necesarios para el buen desarrollo de sus clases. Sobresale el trabajo en equipo, las discusiones guiadas sobre conceptos o definiciones de leyes o propiedades, las demostraciones explicativas, la utilización de materiales, sucesos y actividades de su entorno en el proceso de analizar, comprender, implementar y resolver las situaciones que se les presentan.

Además, y lo más importante, es que esta propuesta no se basa simplemente en "enseñar a comprender", sino que va más allá, pues se fundamenta en la orientación de "enseñar a enseñar". 


\section{ESQUEMAS REPRESENTATIVOS DE LA PROPUESTA}
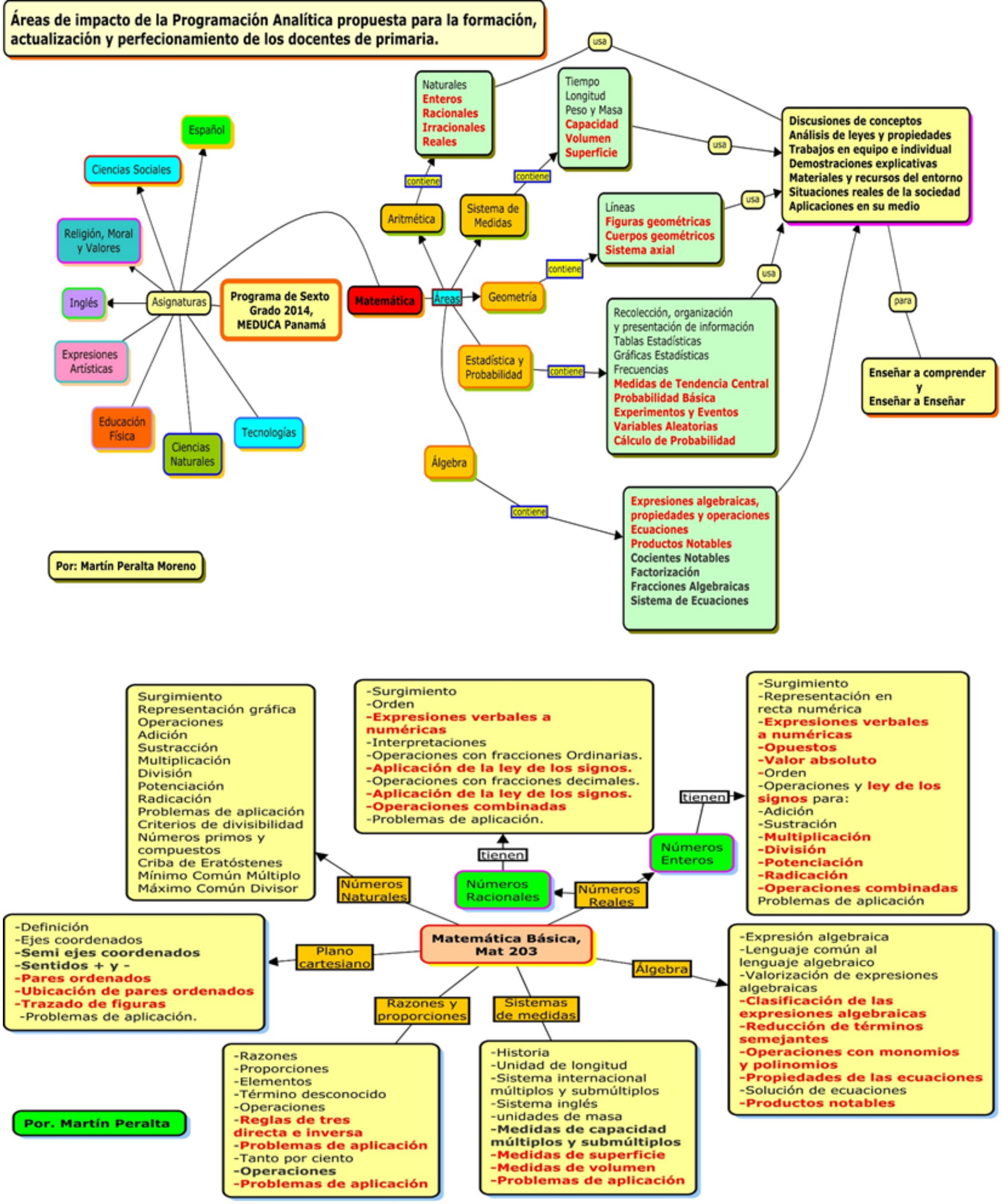

Propuesta para la complementación de los contenidos de la asignatuara Matemática Básica o Mat 203 de la Licenciatura en Educación Primaria 


\section{CONCLUSIONES}

En este trabajo se presenta la formación en matemáticas modernas que reciben los docentes egresados de la Licenciatura en Educación Primaria como un campo de acción e investigación de la evaluación y perfeccionamiento docente, que tiene especial relevancia por la importancia decisiva de la función docente como catalizadora y gestora de los aprendizajes. Cada una de las cuestiones abordadas en este trabajo y los recursos que se proponen están abiertos a su experimentación, evaluación y mejora progresiva.

La formación matemática de los maestros es una preocupación relevante a nivel nacional e internacional. Esta preocupación se genera al reconocer que el conocimiento de matemáticas de los maestros es un elemento clave para la mejora de la enseñanza.

En este sentido, a partir de la situación planteada y en función de esta investigación se concluyó dándole respuestas específicas a los objetivos, en ese orden se obtuvo que:

$>$ Los docentes informantes tienen grandes dificultades en el diseño, ejecución y explicación de las temáticas concernientes a las matemáticas modernas, lo cual puede deberse a que su formación académica no fue lo suficientemente completa e integral en la asignatura de matemáticas, ya que sus dominios, tanto conceptuales como procedimentales, están modestamente desarrollados.

$>$ En el desarrollo de las clases de matemáticas, los docentes utilizan escasas estrategias de enseñanza, ya que prevalece la exposición magistral en el tablero por parte del maestro, el trabajo individual en clase y las tareas o prácticas resueltas en el cuaderno por los estudiantes. En ninguna de las clases de matemática se observó la utilización de las tecnologías como recurso de apoyo educativo.

$>$ Los educadores estiman que es necesario hacer ajustes en los contenidos y en la enseñanza que se ofrece en los cursos universitarios de matemáticas en la Licenciatura de Educación Primaria, ya que estos no les ayudaron a adquirir destrezas ni a dominar los elementos propios de cada área de la asignatura, y menos ahora con la inclusión de nuevos contenidos en los programas curriculares del Ministerio de Educación. Esta débil formación en matemáticas los lleva a presentar una actitud de resistencia y poca motivación por enseñar las matemáticas modernas.

El plan de estudios de los diferentes centros formadores de docentes de primaria contemplados en esta investigación muestran una malla curricular con pocas asignaturas referentes a matemáticas, ya que las materias y los créditos alcanzan un promedio de $6.5 \%$ del total, lo cual quiere decir que los participantes no reciben gran cantidad de horas de estudio y preparación en las diferentes áreas de las matemáticas que luego deben 
desarrollar en las aulas de clases.

$>$ Las acciones de mejoras que se incorporan en el programa de formación, actualización y perfeccionamiento profesional para los docentes de primaria en el área de las matemáticas modernas son dos; la primera está constituida por la Programación Analítica de dos cursos o seminario-taller necesarios para la actualización y perfeccionamiento profesional de los docentes en servicio sobre las áreas de las matemáticas modernas contempladas en los nuevos programas curriculares; la segunda consiste en la reestructuración del Diseño Curricular de los cursos Matemática I y II, que constituyen las asignaturas del plan de estudio de la Licenciatura en Educación Primaria que se imparte en las instituciones formadoras de docentes, complementándolas con las áreas y los contenidos de las matemáticas modernas.

> Se implementó, del 6 al 10 de junio de 2016, del programa seminario-taller: "Estrategias Metodológicas para la Enseñanza de los Números Enteros y del Álgebra a Nivel Primario" en la Escuela Octavio Méndez Pereira, con una participación de 14 de los 23 docentes que hay en la educación primaria.

$>$ Se pudo evaluar el alcance e impacto que tuvo desarrollo del seminario en los educadores participantes de dos maneras; la primera por la comparación de los resultados obtenidos en las oportunidades con la prueba o pre-test, en donde los resultados de la segunda oportunidad fueron significativamente mucho mejor que en la primera oportunidad; la segunda fue a través de la evaluación comentada y escrita que aplicó las autoridades del Ministerio de Educación, en donde se reflejaba el grados de satisfacción por la participación en el mismo.

Por lo anterior expuesto, es razonable e imperativo buscar alternativas para actualizar y modificar el sistema de enseñanza-aprendizaje de las matemáticas en todas sus áreas y en todos los niveles del sistema educativo, así como reorientar la formación del docente de primaria tras una consensuada política de estado.

\section{REFERENCIAS BIBLIOGRÁFICAS}

- Álvarez, C. (2008). La etnografía como modelo de investigación en educación. Facultad de Ciencias de la Educación. Universidad de Oviedo, Oviedo. España.

- Andrew M, McKinsey . (2007). Cómo hicieron los sistemas educativos con mejor desempeño del mundo para alcanzar sus objetivo. McKinsey \& Company .

- Ausubel, D.P.; Novak, J.D. (1983). Psicología educativa: un punto de vista cognoscitivo. México, Editorial Trillas. Traducción al español, de Mario Sandoval P., de la segunda 
edición de Educational psychology : a cognitive view.

- Beltrán, U; Cruz, Alejandro. (2014). Mexicanos ven a maestros mal preparados. México. http://www.excelsior.com.mx/nacional/2014/05/19/960096

- Cáceres, D. (2014). "Mal desempeño docente refleja pésimo rendimiento de los alumnos". Dirección General de Evaluación de la Calidad Educativa, Honduras.

- Cardelli, J; Duhalde, M. ( ). Formación Docente en América Latina. Una Perspectiva Político-Pedagógica. UNESCO - OEI.

- http://www.oei.es/docentes/articulos/formacion_docente_AL_perspectiva_politico_ pedagogica.pdf

- Cardelli, J. (1999): "Reflexiones críticas para una política nacional de formación docente”, Cuadernos de Educación, Serie Formación Docente, Año 1, Nro. 2, CTERA, Buenos Aires.

- Castillo, N. (2007). Informe de Progreso Educativo de Panamá.

- http://www.empresariosporlaeducacion.org/sites/default/files/5_informepanama2007. pdf

- Castillo, N. (2012). Políticas docentes en Centroamérica. Tendencias nacionales. Panamá. Universidad de Panamá.

- Castro, E. (Ed.) (2001). Didáctica de la matemática en la educación primaria.

- Madrid, España.

- Collado, W. (2015). "Liderazgo y escuelas efectivas: cómo lograr instituciones educativas con sinergia y alto rendimiento”. Santo Domingo, República Dominicana.

- Cutimbo, P. (2008). "Influencia del nivel de capacitación docente en el rendimiento académico de los estudiantes del instituto superior pedagógico público de Puno: caso de la especialidad de educación primaria”. Lima, Perú.

- http://cybertesis.unmsm.edu.pe/bitstream/cybertesis/2395/1/Cutimbo_ep.pdf

- Denzin, N. (1978): The research act. A theoretical introduction to sociological methods, Editorial Mc Graw Hill, New York

- Díaz B., F (2002). Aportaciones de las perspectivas constructivista y reflexiva en la formación docente. Perfiles educativos 24 (97-98): 6-25.

- Díaz B, F. (2010). Estrategias docentes para un aprendizaje significativo: una interpretación constructivista. México: McGraw-Hill.

- Durán, D. (2015). La matemática no se enseña, se aprende. Venezuela.

- http://www.viceacademico.luz.edu.ve/index.php?option=com_content\&task=view\&id= 1069\&Itemid $=202$

- Falus, L; GOLDBERG.(2011). Perfil de los docentes en América Latina. OEI. UNESCO. Buenos Aires, Argentina. 
- http://www.siteal.iipe-oei.org/sites/default/files/cuaderno09_20110624.pdf

- Gaceta Oficial. (2004). "Por el cual se aprueba el texto único de la Ley 47 de 1946, Orgánica de Educación, con numeración corrida y ordenación sistemática conforme fue dispuesto por el artículo 26 de la Ley 50 de 1 de noviembre de 2002”. Panamá.

- Galtung, J. (1973). Teoría y método de la investigación social. Eudeba, Buenos Aires, Argentina.

- García, G. ( ). Estándares Básicos de Competencias en Matemática. Ministerio de Educación de Colombia.

- http://www.mineducacion.gov.co/1621/articles-116042_archivo_pdf2.pdf

- Godino, J. D. (2002a). Investigaciones sobre teoría de la educación matemática.

- URL: http://www.ugr.es/local/jgodino/teoria.htm/.

- Godino, J. D. (2002b). Investigaciones sobre el significado y comprensión de las objetos matemáticos.

- URL: http://www.ugr.es/local/jgodino/semiotica.htm

- Godino, J.D (2002). Formación matemática y didáctica de maestros. Departamento de Didáctica de la Matemática. Universidad de Granada, España.

- Godino, J. (2004). Didáctica de las matemáticas para maestros.

- $\quad$ Universidad de Granada, España.

- Hernández, C (1999). II Seminario Taller sobre perfil del docente y estrategias de formación. El Salvador.

- La Prensa. (2012). Enseñanza de matemática en escuelas tiene fallas. Universidad Tecnológica de Panamá. Panamá.

- http://impresa.prensa.com/panorama/Ensenanza-matematica-escuelasfallas_0_3775122601.html

- Lacarriere, J. (2008). Tesis doctoral titulada "La formación docente como factor de mejora escolar"

- https://repositorio.uam.es/bitstream/handle/10486/1760/11829_lacarriere_espinoza. pdf?sequence $=1$

- Lebrija, A; Flores, R; Trejos, M. (2008). El papel del maestro, el papel del alumno: un estudio sobre las creencias e implicaciones en la docencia de los profesores de matemáticas en Panamá. Panamá.

- López, R. (2013). Enseñanza de matemática en escuelas tienen falla. Informe de la UTP Panamá, 2012.

- Martínez, P. (2006). El método de estudio de caso. Estrategia metodológica de la investigación científica. España.

- http://www.redalyc.org/pdf/646/64602005.pdf 
- MEDUCA-PRODE. (2005). Perfil del nuevo docente panameño. Panamá.

- Mella, O. (2000). Técnicas de investigación cualitativas, grupos focales. CIDE, Santiago, Chile, 2000

- http://biblioteca.uahurtado.cl/ujah/856/txtcompleto/txt105091.pdf

- Morales, V. (1998). Sobre la construcción de teorías: o hacer ciencia es algo más que investigar. CEISEA. Caracas, Venezuela.

- Morin, E. (2001). Los siete saberes necesarios para la educación del futuro. UNESCO. México.

- Muñiz, M. ( ). Estudios de casos en la investigación cualitativa. Universidad Autónoma de Nuevo León, México.

- http://www.psico.edu.uy/sites/default/files/cursos/1_estudios-de-caso-en-lainvestigacion-cualitativa.pdf

- Ochoa, S. (2015). Informe Nacional sobre Docentes para la Educación de la Primera Infancia: PERÚ. UNESCO-OREALC, Lima, Perú. .

- OEI; CECC. (1999). Establecimiento de estándares para la educación primaria en Centroamérica. Panamá.

- OEI-OPREAL-COSPAE. (2002). Informe de Progreso Educativo de Panamá.

- Parica R, A; Bruno L, F; Abancin O, R. (2005). Teoría del constructivismo social de Lev Vygotsky en comparación con la teoría Jean Piaget. Universidad Central de Venezuela. Venezuela. http://constructivismos.blogspot.com/

- Rodríguez, G; Gil, J; García, E. (1996). Metodología de la investigación cualitativa.

- Granada. España.

- Salgado, A. (2007). Investigación cualitativa: diseño, evaluación del rigor metodológico y retos. Universidad de San Martín de Porres. Lima, Perú.

- http://www.scielo.org.pe/scielo.php?script=sci_arttext\&pid $=$ S1729-48272007000100009

- Tenti, E. (1988). "El oficio del maestro. Contradicciones iniciales", en Maestros. Formación, práctica y transformación escolar, Miño y Dávila Editores, Buenos Aires.

- Toffler, A. (1970).

- Toffler, A. (1983). La Tercera Ola, Plaza y Janés, México.

- Toffler, A; Toffler, H. (1995). La creación de una nueva civilización: La política de la tercera ola, Plaza y Janés, México.

- Torres, R. M. (1996): “Formación docente: Clave de la reforma educativa", en Nuevas formas de enseñar y de aprender. Santiago, Chile.

- Sadovsky, P. (2010). La enseñanza de la matemática en la formación docente para la 
escuela primaria. Buenos Aires, Argentina.

- Sampieri, R. (2013). El Marco Teórico. Universidad Celaya.

- https://www.youtube.com/watch?v=TH9YF3Y2GDE\&1ist=PLsBeaHOvaMJRMcN5y0C6nwdbt5O5HPUI

- Sánchez, H. (2004). "La formación inicial de los maestros de educación primaria: Un análisis comparativo de las propuestas de formación docente en Argentina, Costa Rica, Chile y México". México, D.F.

- http://www.bib.uia.mx/tesis/pdf/014443/014443.pdf

- UNESCO. (2003). ¿Cómo estamos formando a los maestros en América Latina?. Editora: Isabel Flores. Perú.

- UTP Panamá. (2012). Informe sobre la enseñanza de las matemáticas en las ecuelas primarias de Panamá. Panamá.

- Vásquez, C. (2014). "Evaluación de los conocimientos didáctico-matemáticos para la enseñanza de la probabilidad de los profesores de educación primaria en activo". Universitat de Girona. España.

- http://dugi-doc.udg.edu/bitstream/handle/10256/9749/tcavo.pdf?sequence=1

- Weiner, B. (1985): An attributional theory of achievement motivation and emotion. Psychological Review, 92, 548 $\neg 573$.

- Weiner, B. (1986): An atributional theory of motivation and emotion. New York: SpringerVerlag. 